\title{
Synthesis of 1,3,4-oxadiazoles derivatives from a-amino acids and acyl hydrazides using microwave irradiation
}

\section{Bruna Simões Martins, ${ }^{\mathrm{a}^{*}}$ Elisiane Frantz Heck, ${ }^{\mathrm{a}}$ Caroline Raquel Bender, ${ }^{\mathrm{a}}$ Wolmar A. Severo Filho, ${ }^{b}$ Antonio L. Braga, ${ }^{c}$ Oscar E. Dorneles Rodrigues, ${ }^{a}$ Luciano Dornelles ${ }^{a}$}

\author{
${ }^{a}$ Departamento de Química, Universidade Federal de Santa Maria, 97105-900, Santa Maria, RS, Brazil \\ ${ }^{b}$ Departamento de Química e Física, Universidade de Santa Cruz do Sul, Santa Cruz do Sul, RS, Brazil \\ ${ }^{c}$ Departamento de Química, Universidade Federal de Santa Catarina, Florianópolis, SC, Brazil \\ * Corresponding author. Tel.: +55(55)32208669; bruna.simoesm@gmail.com
}

\section{Keywords: amino acids; 1,3,4-oxadiazoles; microwave irradiation}

\section{INTRODUCTION}

The 1,3,4-oxadiazoles constitute an important family of heterocyclic compounds as they have attracted significant interest in medicinal chemistry, pesticide chemistry and polymer science. These compounds derivatives have been found to exhibit diverse biological activities such as analgesic, ${ }^{1}$ antiinflammatory, ${ }^{2}$ antimicrobial, ${ }^{3}$ anti-HIV ${ }^{4}$ and other biological properties. Consequently, the synthesis of compounds containing this heterocycle core has attracted considerable attention and a wide variety of methods have been used for its assembly. By far the most common synthetically protocol involves the dehydrative cyclization of diacylhydrazides, usually with strongly acidic reagents such as $\mathrm{SOCl}_{2},{ }^{5} \mathrm{P}_{2} \mathrm{O}_{5}$, $\mathrm{POCl}_{3}{ }^{7}$ and $\mathrm{H}_{2} \mathrm{SO}_{4}{ }^{8}$.

\section{RESULTS AND DISCUSSION}

We now describe herein an improved procedure for the preparation of 1,3,4-oxadiazoles 3a-e (Scheme $1)$, using microwave irradiation. The oxadiazoles were obtained in good yields (54-75\%), from acyl hydrazides 1a-c and N-protected $\alpha$-amino acids 2ae in presence of $\mathrm{POCl}_{3}$.

Scheme 1

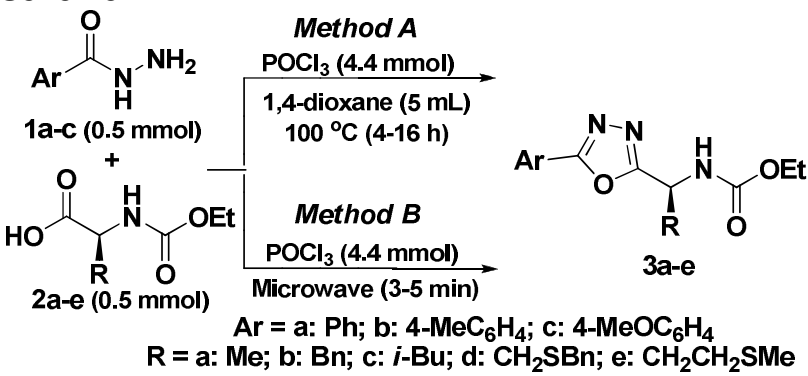

The reaction tube was placed inside the cavity of a CEM Discover focused microwave synthesis system, operated at $100 \pm 5{ }^{\circ} \mathrm{C}$, power $200-250 \mathrm{~W}$. The products were isolated and characterized by spectroscopic methods ( ${ }^{1} \mathrm{H}$ NMR and ${ }^{13} \mathrm{C}$ NMR). The respective compounds 3 were also obtained using conventional heating in an oil bath $100^{\circ} \mathrm{C}$ temperature, but requires an extended period of time, 4-16 h. Table 1 shows comparison of eleven conventionally prepared oxadiazoles 3a-e with the ones obtained by microwave procedure.

Table 1. Comparison of conventional heating and microwave irradiation in the synthesis of $1,3,4$-oxadiazoles 3.

\begin{tabular}{|l|c|c|c|c|}
\hline Entry & \multicolumn{2}{|c|}{ Conventional method } & \multicolumn{2}{c|}{ Microwave method } \\
\hline Compounds & Time (h) & $\begin{array}{c}\text { Yield } \\
(\%)\end{array}$ & $\begin{array}{c}\text { Time } \\
(\mathrm{min})\end{array}$ & $\begin{array}{c}\text { Yield } \\
(\%)\end{array}$ \\
\hline 3aa & 7 & 51 & 4 & 62 \\
\hline 3ab & 4 & 57 & 3 & 65 \\
\hline 3ac & 7 & 41 & 4 & 70 \\
\hline 3ad & 7 & 40 & 4 & 54 \\
\hline 3ae & 4 & 58 & 3 & 72 \\
\hline 3ba & 16 & 45 & 4 & 63 \\
\hline 3bb & 16 & 40 & 4 & 75 \\
\hline 3bc & 16 & 41 & 4 & 60 \\
\hline 3ca & 4 & 40 & 4 & 56 \\
\hline 3cc & 16 & 40 & 4 & 54 \\
\hline 3ce & 16 & 42 & 5 & 60 \\
\hline
\end{tabular}

\section{CONCLUSION}

In conclusion we have developed a one-pot synthesis of 2,5-disubstituted-1,3,4-oxadiazoles under microwave irradiation using phosphorous oxychloride as an efficient promoter. Good yields, short reaction times, simple operation and easy work-up procedure are some advantages of this protocol compared to their synthesis under conventional thermal heating conditions.

\section{ACKNOWLEDGEMENTS}

CNPq, CAPES.

\section{REFERENCES}

${ }^{1}$ Akhter, M.; Husain,A.; Azad,B.; Ajmal,M. Eur. J. Med. Chem., 2009,44, 2. Jayashankar, B.; Lokanath Rai, K.M.; Baskaran, N.; Sathish, H.S. Eur. J. Med. Chem., 2009, 44, 3898.

${ }^{3}$ Gupta, V.; Kashaw, S.K.; Jatav, V.; Mishra, P. Med. Chem. Res., 2008, $17,205$.

${ }^{4}$ El-Emam, A.A.; Al-Deeb, O.A.; Al-Omar, M.; Lehmann, J. Bioorg. Med Chem., 2004, 12, 5107.

${ }^{5}$ Brog, S.; Vollinga, R. C.; Labarre, M.; Payza, K.; Luthman L.; Terenius, K. J. Med. Chem., 1999, 42, 4331.

6 Shafiee, A.; Naimi, E.; Mansobi, P.; Foroumadi A.; Shekari, M. J. Heterocycl. Chem., 1995. 32, 1235

${ }^{7}$ He, D.-H.; Zhu, Y.-C.; Yang, Z.-R.; Hu A.-X.; Cao, G. Turk. J. Chem., 2009, 33, 1

${ }^{8}$ Sharma,S.; Srivastava,V.K; Kumar, A. Eur. J. Med. Chem., 2002, 37, 689. $14^{\text {th }}$ Brazilian Meeting on Organic Synthesis - $14^{\text {th }}$ BMOS - September 01-05, 2011-Brasilia, Brazil 